\title{
Study of Effect on the Performance and Composite Reinforcement Mechanism about Short Steel Fiber and Vehicle Tire Tread
}

\author{
Qi Xiao-jie ${ }^{1, a}$, Wang Qiang ${ }^{2, b}$, Wang Wei, ${ }^{3, c}$, Yang Zhao ${ }^{4, d}$ \\ 1,2,3,4 HeiLongjiang Institute of Technology, HarBin, China \\ ${ }^{2}$ Northeast Forestry University, HarBin, China

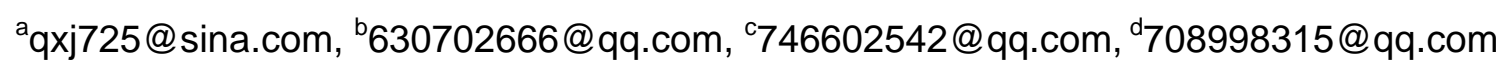

Keywords: short steel fiber; vehicle tire tread rubber; strengthening mechanism; effect on the performance

\begin{abstract}
Short steel fiber composite tire tread rubber can greatly combine rigidity of short steel fiber with flexibility of tire tread rubber, which can considerably improve performance of tire. Tire tread used in heavy load vehicles or engineering vehicles has problems of easily cracking, chunking, chipping, quickly wearing and so on under the severely working condition. In order to effectively deal with the problems mentioned above, short steel fiber is used as strengthening substance and tire tread rubber is used as base material through processing surface of short steel fiber with copperizing way. Thus composite formula system and blending technique of short steel fiber and vehicle tire tread can be designed. Physical model of composite strengthening blending of short steel fiber and tire tread composite reinforcement dispersing model under the condition of four sporadic types of short steel fiber can be constructed. Then composite reinforcement mechanism of short steel fiber and tread rubber is explored from the two processes of physics and chemistry, and efficient combination of short steel fiber and tire tread rubber is realized. A contrast experiment on stretching strength, tearing strength, wearing index, hardness and dent resistance rebound resilience of different contents of short steel fiber is carried, so performance effect rules of short steel fiber to tread rubber is achieved. The results show that distribution of short steel fiber in tread rubber has an optimal content value which is the ideal one of reinforcement effect and the performance of composite base is greatly improved, when steel fiber content is about $10 \%$.
\end{abstract}

\section{Introduction}

Composite material of short steel fiber strengthening rubber is to combine rigidity of short steel fiber with flexibility of rubber which keeps high elasticity of rubber and effectively improve rubber modulus. Short steel fiber strengthens tire tread and makes it get good performances of high modulus, high rigidity, cutting proof, tearing proof, piercing proof, heavy load proof and fatigue proof, which becomes main technical means to realize high performance of tire rubber material. Tire tread usually is pierced, chunked, or chipped, when tires work under the severe conditions, for example: tires of heavy load vehicles and engineering vehicles often work on mining area with many sharp sandstone. During work, tire tread is usually pierced, chunked or chipped, which considerably influences its life expectancy. This paper takes short steel fiber as reinforcement substance to realize the effective composite of it and tire tread rubber by plating technique and explores composite reinforcement mechanism of short steel fiber and tire tread rubber through analyzing performance. Thus this can provide theory basics for manufacturing high performance tire [1-2].

Project source: Youth Found of Heilongjiang Institute of Technology (2012QJ07)

\section{Composite Reinforcement Mechanism Study on Short Steel Fiber and Tread Rubber}

(1)Physical Model of Short Steel Fiber and Tread Rubber Composite Reinforcement Blending

Two complementary and equivalent processes are used to describe composite reinforcement blending physical model of short steel fiber and tread rubber. The first process is that short steel fiber 
is hypothetically considered as many bar-shaped object; during blending, short steel fiber blended is squeezed into rubber sizing to form composite reinforcement physical model which takes rubber sizing as main framework, and this process is shown in Fig. 1(a). The second process is that spaces among short steel fiber are hypothetically considered as many tubules or capillaries, during blending, rubber sizing is squeezed into tubules and capillaries to form composite reinforcement physical model which takes short steel fiber as main framework, and this process is shown in Fig.1(b). Whether blending smelting of rubber sizing and short steel fiber is completed successfully or not depends on viscosity of rubber sizing. The less viscosity of rubber sizing is, the more easily short steel fiber is squeezed into rubber sizing or rubber sizing is squeezed into space among short steel fiber, vice verse[3-4].

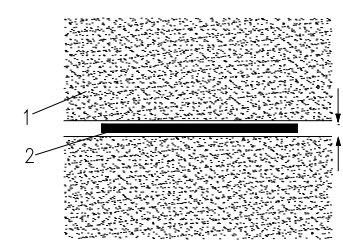

1-rubber sizing

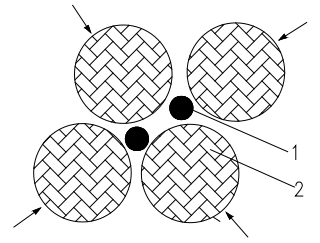

2- short steel fiber

(a)the model taking rubber sizing as framework $\quad$ (b)the model taking short steel fiber as framework
Fig.1 Composite Reinforcement Blending Physical Model of Short Steel Fiber and Tread Rubber

(2)Composite Reinforcement Dispersing Physical Model of Short Steel Fiber and Tread Rubber

There are four cases about composite reinforcement dispersing physical model of short steel fiber and tread rubber. It is shown in Fig.2, the first is that short steel fiber proportion is high which mainly exists in rubber sizing in form of agglomeration. The second is short steel fiber proportion is low and there is not short steel fiber in rubber sizing at all. The third is that a little bit or few of short steel fiber attaches to the surface of rubber sizing, or exist in form of unbound state. The fourth is that short steel fiber proportion is middle which evenly disperses in the whole rubber sizing by a good dispersing process.Obviously, the first three situations are that short steel fiber unevenly distributes in rubber sizing, but the fourth state is an ideal condition. Blending smelting rubber sizing needs to be in the state of optimal viscosity to keep short steel fiber blending smelting evenly. When blending smelting sizing is in its best state, short steel fiber blendingly smelted is easy to disperse into rubber sizing. Short steel fiber can get a better dispersing degree taking less time and using less power. Physical model of short steel fiber agglomeration's shattering and dispersing is shown in Fig.3. In the first case, when short steel fiber agglomeration becomes deformed under the action of cutting and stretching, short steel fiber agglomeration can be shattered; in the meantime, with the amount of the deformed increasing, short steel fiber that unevenly disperses and dissociates will increase, and gradually and evenly disperses into rubber sizing[5].


1-short steel fiber agglomeration 2- rubber sizing 3-dissociating short steel fiber
(a) the first case
(b) the second case
(c) the third case (d) the fourth case

Fig.2 composite reinforcement dispersing physical model of short steel fiber and tread rubber

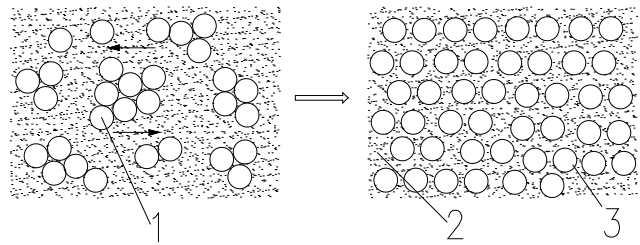

1- short steel fiber agglomeration 2- rubber sizing 3- shattering short steel fiber

Fig. 3 Physical Model of Short Steel Fiber Agglomeration's Shattering and Dispersing

(3)Composite Reinforcement Mechanism Study on Short Steel Fiber and Tread Rubber 
When brass is plated on short steel fiber, the layer CuZn and $\mathrm{ZnO}$ is formed on its surface. $\mathrm{Zn}$ ions disperse towards outside surface under the pulling force, and a stacking layer of $\mathrm{CuO}$ and $\mathrm{ZnO}$ forms, between which two layers mutually disperse. Then a thinner layer of $\mathrm{Cu}_{2} \mathrm{O}$ is formed on the outermost layer of $\mathrm{Zn}$ ions. Surface construction model of short steel fiber plated brass is shown in Fig.4, in which thickness of $\mathrm{CuO}_{2}$ layer is about $2 \mathrm{~nm}, \mathrm{CuO}+\mathrm{ZnO}$ layer is about $10 \mathrm{~nm}, \mathrm{CuZn}+\mathrm{ZnO}$ layer is about $40 \mathrm{~nm}$, the whole plating brass is about $52 \mathrm{~nm}$.
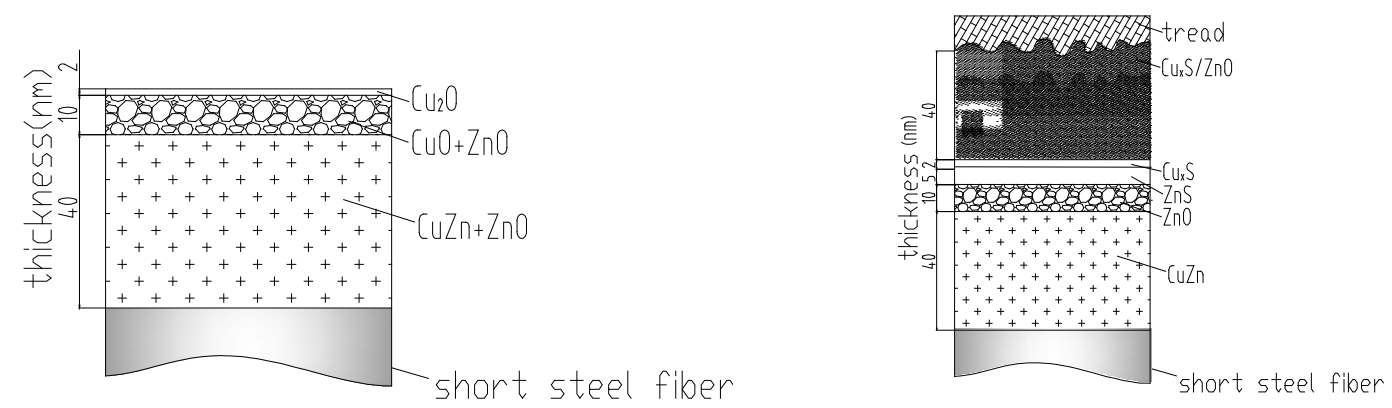

Fig. 4 Surface Construction Model of Short Steel Fiber Plating Brass Fig.5 Construction Model of Short Steel Fiber and Tread Sizing Reinforcement Effect Mechanism

Short steel fiber and tread rubber composite reinforcement are divided two processes. One is a physical process which short steel fiber and tread rubber sizing are intertwined and stuck by thicker C501, which makes short steel fiber forms the framework model of strengthening rib in tread sizing. The other is chemical process of vulcanization. At the beginning of vulcanization action, brass ions(Cu2+), Zn ions ( $\mathrm{Zn2+}$ )and free electrons (e-) of the plated brass short steel fiber form the layer of $\mathrm{CuS}$ and $\mathrm{ZnS}$ by positive ions disperse onto the surface of short steel thread. Because oxidizing electric potential of sub-brass ions(e-) is higher that of $\mathrm{Zn}$ ion ( $\mathrm{Zn2+}), \mathrm{Zn2+}$ ) is more easily to react with sulfur to form $\mathrm{ZnS}$. But the layer of $\mathrm{ZnS}$ is quickly covered by the $\mathrm{CuS}$ layer because of $\mathrm{CuO}$ layer on the surface of short steel fiber. The CuS layer can effectively improve sticking strength between short steel fiber and tread rubber sizing, when the CuS layer permeates into tread rubber sizing and is bond by it. Rubber sizing and short steel fiber are sticken by sulfide linkage to form sticking layer whose bonding force surpasses that of rubber itself, and acts as a good reinforcement effect. The construction model of reinforcement effect mechanism is shown in Fig.5, in which thickness of CuS layer has a optimal value that is generally $2-4 \mathrm{~nm}$. The thickness is neither too thick nor too thin, because if it is too thick to drop and too thin to mutually permeate with rubber material effectively, resulting in decreasing of sticking strength[6-7].

\section{Performance Effect Analyses That Short Steel Fiber Strengthens Tire Tread}

Tab. 2 Physical Mechanical Performance Testing Results of Tread Rubber

\begin{tabular}{|c|c|c|c|c|}
\hline Testing items & $\mathrm{TR}_{0}$ & $\mathrm{TR}_{5}$ & $\mathrm{TR}_{10}$ & $\mathrm{TR}_{20}$ \\
\hline Stretching strength (MPa) & 23.5 & 26.8 & 31.6 & 30.7 \\
\hline Elasticity modulus (MPa) & 6.75 & 8.65 & 10.91 & 14.23 \\
\hline Rupture stretching ratio (\%) & 594 & 516 & 478 & 420 \\
\hline Tearing strength $(\mathrm{MPa})$ & 19.6 & 22.5 & 25.1 & 28.7 \\
\hline Akronwearing index & 0.28 & 0.31 & 0.35 & 0.33 \\
\hline A type Shao Er hardness (degree ) & 63 & 65 & 68 & 69 \\
\hline shockproof and resilience (\%) & 6 & 9.5 & 12.5 & 18 \\
\hline Sticking strength of short steel fiber and tread $(\mathrm{kN} / \mathrm{m})$ & 1 & 41 & 43 & 41 \\
\hline
\end{tabular}

Note; $\mathrm{T}_{0}$ represents common tread rubber; $\mathrm{T} \mathrm{R}_{5}$ represents tread rubber containing $5 \%$ short steel fiber; $\mathrm{T} \mathrm{R}_{10}$ represents tread rubber containing $10 \%$ short steel fiber; $\quad \mathrm{T} \mathrm{R}_{20}$ represents tread rubber containing $20 \%$ short steel fiber

Obvious changes of physically mechanical performance of tread rubber base have taken place, if short steel fiber of over 5\% content fraction is put in it shown in Tab.2. Stretching strength, elasticity modulus, tearing strength, Akron wearing index, hardness and resilience all increase with the amount of short steel fiber rising, in which stretching strength improves from 23.5MPa to 31.6MPa, improving rate is $30 \%$; elasticity modulus rises from $6.75 \mathrm{MPa}$ to $10.91 \mathrm{MPa}$, which is 1.6 time of its original; tearing strength improves 1.3 time; wearing -proof index improves 1.25 time and 
performance of wearing proof greatly increase, when content fraction increases from $5 \%$ to $10 \%$. However, when content fraction gets to $20 \%$, stretching strength and fatigue proof of composite base don't rise and begin to decrease, and elasticity, tearing strength, hardness and shock proof and resilience increase all the time, at point of which elasticity modulus increases over two time, tearing strength increases near $40 \%$, but rupture stretching rate decrease. The content of short steel fiber in tread rubber will considerably influence physically mechanical performance of tread shown above. The content of short steel fiber has an optical value, when $5 \mathrm{~mm}$-long short steel fiber is used as enhancement object according to formula system of this research, and $10 \%$ content fraction will get optimal reinforcement effect on tread rubber.

\section{Summary}

(1)In this paper, formula system of short steel fiber composite tire tread rubber is designed, and composite reinforcement blending physical model constituted by two complementation and equivalent processes and four kinds of dispersing composite reinforcement scattering physical models are constructed. When tread rubber sizing is under the condition of optimal viscosity degree, dispersing situation of short steel fiber in tread rubber sizing is ideal.

(2)Composite reinforcement mechanism of short steel fiber and tread rubber is analyzed from two aspects of physical and chemical process.

(3)Short steel fiber processed by plating brass can strengthen tire tread rubber, and when the length of short steel fiber is $5 \mathrm{~mm}$, and the content is $10 \%$, its strengthening effect is more obvious and stretching proof and tearing strength considerably increase.

\section{References}

[1] Yu Qingxi. Present situation and development of OTR tires[J].China Rubber/Plastics Technology and Equipment, 2014,01:5-19.

[2] Wang Jinwen.New progress of tire cords and rubber adhesive(one)[J].World Rubber industry, 2012,08:42-47.

[3] Wang Jinwen.New progress of tire cords and rubber adhesive(two)[J].World Rubber industry, 2012,09:26-31.

[4] Zhao Min. Pad and method for large engineering tyre prevulcanization in the preparation method[J]. tire industry, 2011,10:623.

[5] Ding Chen, Liang Jicai, Yin Yanning. The enhancement of surface treatment technology of rubber to metal bonding materials [J].Synthetic Rubber Industry, 2013,05:405-410.

[6] Zheng Hui, Ceng Fanwei. The rubber and metal material adhesion strength influence factors[J]. Rubber Industry,2013,11:697-701.

[7] June. Bonding properties and mechanism of natural rubber and copper wire[D].Qingdao University of Science \& Technology,2012. 